\title{
A spectral collocation method for stochastic Volterra integro-differential equations and its error analysis
}

\author{
Sami Ullah Khan ${ }^{1}$, Mushtaq Ali ${ }^{2}$ and Ishtiaq Ali ${ }^{1,3^{*}}$
}

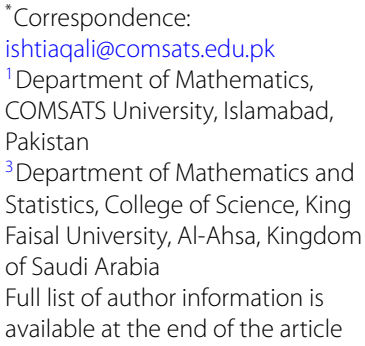

${ }^{3}$ Department of Mathematics and Statistics, College of Science, King Faisal University, Al-Ahsa, Kingdom of Saudi Arabia

Full list of author information is

available at the end of the article

\section{空 Springer}

\begin{abstract}
Volterra integro-differential equations arise in the modeling of natural systems where the past influence the present and future, for example pollution, population growth, mechanical systems and financial market. Furthermore, as many real-world phenomena are subject to perturbations or random noise, it is natural to move from deterministic models to stochastic models. Generally exact solutions of such models are not available and numerical methods are used to obtain the approximate solutions. Therefore the efficiency and long-term behavior of approximate solutions for these systems is an important area of investigation. This paper presents a new numerical approach for the approximate solution of stochastic Volterra integro-differential (SVID) equations based on the Legendre-spectral collocation method. In order to fully use the properties of orthogonal polynomials, we use some function and a variable transformation to change the given SVID equation into a new equation, which is defined on the standard interval $[-1,1]$. For the evaluation of the integral term efficiently a Legendre-Gauss quadrature formula will be used. A rigorous error analysis of the proposed scheme will be provided under the assumption that the solution of the given SVID is sufficiently smooth. For the illustration of our theoretical results a number of numerical experiments will be performed.
\end{abstract}

Keywords: Spectral collocation method; Stochastic Volterra integro-differential equations; Legendre-Gauss-Lobatto points; Error analysis

\section{Introduction}

Stochastic Volterra-integral equations arise in mathematical modeling of many physical phenomena such as mechanics, medical, finance, reactor dynamics, etc. These models also arise in the study of biological populations growth model, the automatic systems theory in delay-differential equations and in the investigation of the behavior to more practical dynamical systems in physics and engineering [1-8]. Most particularly, such systems rely on additive noise, governed by certain probability laws, like Gaussian white noise, so it is natural to use the stochastic differential equations or stochastic Volterra-integral equations and stochastic integro-differential equations for most complicated cases [4, 9]. Therefore the study of stochastic differential equations is an important area of research. The explicit solutions of such a type of differential equations are very rare and one must adopt

(c) The Author(s) 2019. This article is distributed under the terms of the Creative Commons Attribution 4.0 International License (http://creativecommons.org/licenses/by/4.0/), which permits unrestricted use, distribution, and reproduction in any medium, provided you give appropriate credit to the original author(s) and the source, provide a link to the Creative Commons license, and indicate if changes were made. 
a numerical technique to solve such problems. Recently, for the simulation of complex or smooth physical phenomena, spectral methods have emerged as a very powerful and efficient numerical technique, which are a well-known class of numerical methods for the solutions of various differential equations due to the spectral rate of convergence [10-12]. These methods for the numerical solutions of stochastic fractional differential equations were most recently used by Raffaele D'Ambrosio et al. [13-15], while Jacobi polynomials for the numerical solution of time fractional diffusion systems was used in $[16,17]$. The aim of this research work is to develop the spectral collocation method using LegendreGauss-Lobatto points for the approximate solution of SVIDEs. For this purpose, let us consider the stochastic Volterra integro-differential equation with convolution kernels of the form [18]

$$
\left\{\begin{array}{l}
d y(t)=f\left(y(t), \int_{0}^{t} G(t-s) y(s) d s\right) d t+g\left(y(t), \int_{0}^{t} H(t-s) y(s) d s\right) d W(t) \\
y(0)=y_{0}
\end{array}\right.
$$

where $W(t)$ is a stochastic Brownian motion, $f, g, G$ and $H$ are known functions, and we consider the two functions $f$ and $g$ to be globally Lipschitz continuous and the linear growth condition is satisfied, which means that there exist positive constants $K_{1}$ and $K_{2}$ for all $x, \bar{x}, t, \bar{t} \in R$ such that

$$
\begin{aligned}
& |f(x, t)-f(\bar{x}, \bar{t})|^{2} \vee|g(x, t)-g(\bar{x}, \bar{t})|^{2} \leq K_{1}\left(|x-\bar{x}|^{2}+|t-\bar{t}|^{2}\right), \\
& |f(x, t)|^{2} \vee|g(x, t)|^{2} \leq K_{2}\left(1+|x|^{2}+|t|^{2}\right) .
\end{aligned}
$$

The notation $\vee$ denotes the maximum one in both. Next, we consider that $G$ and $H$ are continuous and satisfy the following for a positive constant $K_{3}$ :

$$
\|G(t)-G(s)\| \vee\|H(t)-H(s)\| \leq K_{3}|t-s|, \quad t, s \in[0, T],
$$

which implies that

$$
\|G(t)\| \vee\|H(t)\| \leq M<\infty
$$

for any positive constant $M$. The conditions given in Eq. (2)-Eq. (4) can guarantee that Eq. (1) has a unique solution $y(t)$. Since most stochastic differential equations cannot be solved analytically, a number of numerical method are applied to obtain the approximate solutions of stochastic ordinary differential equations, such as the stochastic Runge-Kutta method, the stochastic linear multistep method and the stochastic Taylor method. For more details we refer the reader to $[19,20]$. In contrast, for the approximate solution of SVIDEs, there are very few results in the existing literature. In [21] one studied the convergence of the Euler-Maruyama scheme for SVIDEs under some different assumptions and the analytical and numerical asymptotic stability has been considered for a linear stochastic functional differential equations in [22], which usually apply low-order methods. We will apply spectral methods which are the most accurate and efficient available numerical techniques for the approximate solution of SVIDEs.

The rest of the paper is organized as follows: A brief description of the proposed method is given in Sect. 2. Comprehensive error analyses of the scheme are performed in Sect. 3. 
Numerical experiments are performed in Sect. 4, which illustrate the theoretical results, and a conclusion is given in Sect. 5 .

\section{Description of the spectral collocation method}

Let $\Lambda_{N}=\left\{t_{k}\right\}_{k=0}^{N}$ denote the grid points, which is the space of real polynomials of degree not greater than $N$, and the set of $(N+1)$ LGL points lies in the interval $[-1,1]$. We consider Eq. (1) in the following simplified form [23]:

$$
\begin{aligned}
& d y(t)=y(t) d t+\left(\int_{0}^{t} G(t-s) y(s) d s\right) d t+y(t) d W(t)+\left(\int_{0}^{t} H(t-s) y(s) d s\right) d W(t), \\
& y(0)=y_{0},
\end{aligned}
$$

where $G(s, t)$ and $H(s, t)$, for $s, t \in[0, T]$, are given functions, and $y(t)$ is an unknown stochastic process. Also $W(t)$ is a Brownian motion process, and $y_{0}$ is the initial condition. In order to solve the SVIDE Eq. (6), we first apply the integral on both sides from $\left[0, t_{i}\right]$, which gives

$$
\begin{aligned}
y\left(t_{i}\right)= & y_{0}+\int_{0}^{t_{i}} y(s) d s+\int_{0}^{t_{i}}\left(\int_{0}^{s} G(s-u) y(u) d u\right) d s \\
& +\int_{0}^{t_{i}} y(s) d W(s)+\int_{0}^{t_{i}}\left(\int_{0}^{s} H(s-u) y(u) d u\right) d W(s) .
\end{aligned}
$$

To analyze the integral of Eq. (7) on a standard interval $[-1,1]$, we take a linear transformation in the form $s=\frac{t_{i}}{2}(\theta+1)$, then Eq. (7) takes the following form:

$$
\begin{aligned}
y\left(t_{i}\right)= & y_{0}+\frac{t_{i}}{2} \int_{-1}^{1} y\left(\frac{t_{i}}{2}(\theta+1)\right) d \theta+\frac{t_{i}}{2} \int_{-1}^{1}\left(\int_{0}^{\frac{t_{i}}{2}(\theta+1)} G\left(\frac{t_{i}}{2}(\theta+1)-u\right) y(u) d u\right) d \theta \\
& +\frac{t_{i}}{2} \int_{-1}^{1} y\left(\frac{t_{i}}{2}(\theta+1)\right) d W(\theta) \\
& +\frac{t_{i}}{2} \int_{-1}^{1}\left(\int_{0}^{\frac{t_{i}}{2}(\theta+1)} H\left(\frac{t_{i}}{2}(\theta+1)-u\right) y(u) d u\right) d W(\theta) .
\end{aligned}
$$

Let $\eta_{i k}=\frac{t_{i}}{2}(\theta+1), k=0,1, \ldots, N$. Now by using $(N+1)$ Gauss-Legendre nodes, the quadrature formula associated with the Legendre weight for the integral equation (8) leads to the semi-discretized spectral equation

$$
\begin{aligned}
y\left(t_{i}\right) \approx y_{0} & +\frac{t_{i}}{2} \sum_{k=0}^{N} y\left(\eta_{i k}\right) \omega_{k}+\frac{t_{i}}{2} \sum_{k=0}^{N}\left(\int_{0}^{\eta_{i k}} G\left(\eta_{i k}-u\right) y(u) d u\right) \omega_{k} \\
& +\frac{t_{i}}{2} \sum_{k=0}^{N} W\left(t_{i k}\right) y\left(\eta_{i k}\right) \omega_{k}+\frac{t_{i}}{2} \sum_{k=0}^{N} W\left(t_{i k}\right)\left(\int_{0}^{\eta_{i k}} H\left(\eta_{i k}-u\right) y(u) d u\right) \omega_{k},
\end{aligned}
$$

where $\omega_{k}$ is the weight function, and $W(t)$ is a Brownian motion process. Once again having the linear transformation $u=\frac{\eta_{i k}}{2}(\theta+1)$, applying to Eq. (9),

$$
y\left(t_{i}\right) \approx y_{0}+\frac{t_{i}}{2} \sum_{k=0}^{N} y\left(\eta_{i k}\right) \omega_{k}+\frac{t_{i}}{2} \sum_{k=0}^{N}\left(\frac{\eta_{i k}}{2} \int_{-1}^{1} G\left(\eta_{i k}-\frac{\eta_{i k}}{2}(\theta+1)\right) y\left(\frac{\eta_{i k}}{2}(\theta+1)\right) d \theta\right) \omega_{k}
$$




$$
\begin{aligned}
& +\frac{t_{i}}{2} \sum_{k=0}^{N} W\left(t_{i k}\right) y\left(\eta_{i k}\right) \omega_{k} \\
& +\frac{t_{i}}{2} \sum_{k=0}^{N} W\left(t_{i k}\right)\left(\frac{\eta_{i k}}{2} \int_{-1}^{1} H\left(\eta_{i k}-\frac{\eta_{i k}}{2}(\theta+1)\right) y\left(\frac{\eta_{i k}}{2}(\theta+1)\right) d \theta\right) \omega_{k}
\end{aligned}
$$

Again by using $(N+1)$ Gauss-Legendre nodes, the quadrature formula associated with the Legendre weight for the integral equation (10), we get

$$
\begin{aligned}
y\left(t_{i}\right) \approx y_{0} & +\frac{t_{i}}{2} \sum_{k=0}^{N} y\left(\eta_{i k}\right) \omega_{k}+\frac{t_{i}}{2} \sum_{k=0}^{N}\left(\frac{\eta_{i k}}{2} \sum_{r=0}^{N} G\left(\frac{\eta_{i k}}{2}(1-\theta)\right) y\left(\frac{\eta_{i k}}{2}(\theta+1)\right) \omega_{r}\right) \omega_{k} \\
& +\frac{t_{i}}{2} \sum_{k=0}^{N} W\left(t_{i k}\right) y\left(\eta_{i k}\right) \omega_{k} \\
& +\frac{t_{i}}{2} \sum_{k=0}^{N} W\left(t_{i k}\right)\left(\frac{\eta_{i k}}{2} \sum_{r=0}^{N} H\left(\frac{\eta_{i k}}{2}(1-\theta)\right) y\left(\frac{\eta_{i k}}{2}(\theta+1)\right) \omega_{r}\right) \omega_{k},
\end{aligned}
$$

where $\omega_{r}$ is the weight function.

Suppose that $Y\left(t_{j}\right) \approx y\left(t_{j}\right)$, and assume that the spectral approximate solution is

$$
y(t) \approx Y(t):=\sum_{j=0}^{N} y\left(t_{j}\right) P_{j}(t), \quad 0 \leq t \leq T,
$$

where $P_{j}(t)$ is Lagrange interpolation polynomial concerned with Gauss-Legendre points $\Lambda_{N}=\left\{t_{k}\right\}_{k=0}^{N}$. To compute $P_{j}(t)$, the efficient way is to express it in terms of the Legendre function; see [24, 25]. Using Eq. (12), the numerical spectral scheme of Eq. (11) takes the following form:

$$
\begin{aligned}
y\left(t_{i}\right) \approx & y_{0} \\
+ & \frac{t_{i}}{2} \sum_{j=0}^{N} Y_{j}\left(\sum_{k=0}^{N} P_{j}\left(\eta_{i k}\right) \omega_{k}\right) \\
& +\frac{t_{i}}{2} \sum_{j=0}^{N} Y_{j}\left(\sum_{k=0}^{N} \sum_{r=0}^{N} \frac{\eta_{i k}}{2} G\left(\frac{\eta_{i k}}{2}(1-\theta)\right) P_{j}\left(\frac{\eta_{i k}}{2}(\theta+1)\right) \omega_{r} \omega_{k}\right) \\
& +\frac{t_{i}}{2} \sum_{j=0}^{N} Y_{j}\left(\sum_{k=0}^{N} W\left(t_{i k}\right) P_{j}\left(\eta_{i k}\right) \omega_{k}\right) \\
& +\frac{t_{i}}{2} \sum_{j=0}^{N} Y_{j}\left(\sum_{k=0}^{N} \sum_{r=0}^{N} W\left(t_{i k}\right) \frac{\eta_{i k}}{2} H\left(\frac{\eta_{i k}}{2}(1-\theta)\right) P_{j}\left(\frac{\eta_{i k}}{2}(\theta+1)\right) \omega_{r} \omega_{k}\right) .
\end{aligned}
$$

Now taking $Y:=\left[Y_{0}, Y_{1}, \ldots, Y_{N}\right]^{T}$ and $C_{N}:=\left[y_{0}, y_{1}, \ldots, \phi_{0}\right]^{T}$, then we obtain a more compatible form of Eq. (13)

$$
Y-\left(B_{1}+B_{2}+B_{3}+B_{4}\right) Y=C_{N},
$$


where the entries of the matrices $B_{1}, B_{2}, B_{3}, B_{4} \in \mathbb{R}^{(N+1) \times(N+1)}$ are given now:

$$
\begin{aligned}
& B_{1}(i, j)=\frac{t_{i}}{2}\left(\sum_{k=0}^{N} P_{j}\left(\eta_{i k}\right) \omega_{k}\right), \\
& B_{2}(i, j)=\frac{t_{i}}{2}\left(\sum_{k=0}^{N} \sum_{r=0}^{N} \frac{\eta_{i k}}{2} G\left(\frac{\eta_{i k}}{2}(1-\theta)\right) P_{j}\left(\frac{\eta_{i k}}{2}(\theta+1)\right) \omega_{r} \omega_{k}\right), \\
& B_{3}(i, j)=\frac{t_{i}}{2}\left(\sum_{k=0}^{N} W\left(t_{i k}\right) P_{j}\left(\eta_{i k}\right) \omega_{k}\right), \\
& B_{4}(i, j)=\frac{t_{i}}{2}\left(\sum_{k=0}^{N} \sum_{r=0}^{N} W\left(t_{i k}\right) \frac{\eta_{i k}}{2} H\left(\frac{\eta_{i k}}{2}(1-\theta)\right) P_{j}\left(\frac{\eta_{i k}}{2}(\theta+1)\right) \omega_{r} \omega_{k}\right) .
\end{aligned}
$$

\section{Error analysis}

In this section, we provide the error analysis of the spectral collocation method for SVIDE. We will particularly determine the spectral accuracy for the numerical solution $y^{N}(t)$ [26]. Let us consider

$$
U_{T, 1}^{N}(t)=I_{T, N} \frac{d}{d t} Y(t)-\frac{d}{d t} I_{T, N} Y(t)
$$

and

$$
\begin{aligned}
U_{T, 2}^{N}(t)= & f\left(y^{N}(t), \int_{0}^{t_{i}} G(t, s) y^{N}(s) d s, t\right)-f\left(I_{T, N} Y(t), I_{T, N} \int_{0}^{t_{i}} G(t, s) Y(s) d s, t\right) \\
& \vee g\left(y^{N}(t), \int_{0}^{t_{i}} H(t, s) y^{N}(s) d s, t\right) \\
& -g\left(I_{T, N} Y(t), I_{T, N} \int_{0}^{t_{i}} H(t, s) Y(s) d s, t\right) .
\end{aligned}
$$

Then we have from Eq. (1)

$$
\begin{aligned}
\frac{d}{d t} I_{T, N} Y(t)= & f\left(Y(t), \int_{0}^{t_{i}} G(t, s) Y(s) d s, t\right) \\
& \vee g\left(Y(t), \int_{0}^{t_{i}} H(t, s) Y(s) d s, t\right)-W_{T, 1}^{N}(t), \quad t \in \Lambda_{N} .
\end{aligned}
$$

Furthermore, let $E^{N}(t)=y^{N}(t)-I_{N, T} Y(t)$, then we have from Eq. (6) and Eq. (16) that

$$
\left\{\begin{array}{l}
\frac{d}{d t} E^{N}(t)=U_{T, 1}^{N}(t)+U_{T, 2}^{N}(t), \quad t \in \Lambda_{N} \\
E^{N}(0)=\Phi(0)-I_{T, N} \Phi(0)=0 .
\end{array}\right.
$$

Lemma 3.1 Using the condition Eq. (17), the following inequality holds:

$$
\left(E^{N}(T)\right)^{2} \leq 2\left\|E^{N}\right\|_{T, N}\left(\left\|U_{T, 1}^{N}\right\|_{T, N}+\left\|U_{T, 2}^{N}\right\|_{T, N}\right) .
$$


Proof Since $E^{N}(0)=0$, multiplying Eq. (17) by $2 E^{N}\left(t_{T, k}^{N}\right) \omega_{T, k}^{N}$ and having the resulting equation summed up, $1 \leq k \leq N$, we obtain

$$
2\left(E^{N}, \frac{d}{d t} E^{N}\right)_{T, N}=2\left(U_{T, 1}^{N}, E^{N}\right)_{T, N}+2\left(U_{T, 2}^{N}, E^{N}\right)_{T, N}
$$

Since $\frac{d}{d t} E^{N}(t) \in P_{N-1}(0, T)$, then using the result, if $\psi_{1}, \psi_{2} \in P_{2 N-1}(0, T)$ then $\left(\psi_{1}, \psi_{2}\right)_{T}=$ $\left(\psi_{1}, \psi_{2}\right)_{T, N}$. Using this, then

$$
2\left(E^{N}, \frac{d}{d t} E^{N}\right)_{T, N}=2\left(E^{N}, \frac{d}{d t} E^{N}\right)_{T}=\left(E^{N}(T)\right)^{2} .
$$

Put Eq. (20) into Eq. (19), and using a Cauchy-Schwartz inequality, we obtain Eq. (18).

Next we estimate, $U_{T, 1}^{N}$. For this purpose, the following lemma is given; one may refer to [27].

Lemma 3.2 For the integers $1 \leq r \leq N$,

$$
\left\|U_{T, 1}^{N}\right\|_{T} \leq c T N^{-r} R_{T, 1}^{r}(Y)
$$

where $R_{T, 1}^{r}(\Phi)$ is finite.

Proof For this purpose, using Lemma 3.1. for integers $1 \leq r \leq N+1$, we have

$$
\left\|I_{T, N} \frac{d}{d t} Y-\frac{d}{d t} Y\right\|_{T} \leq c_{1} T N^{-r} R_{T, 1}^{r}(Y)
$$

Again we have, $0 \leq r \leq N$,

$$
\left\|\frac{d}{d t}\left(I_{T, N} Y-Y\right)\right\|_{T} \leq c_{1} T N^{-r} R_{T, 1}^{r}(Y)
$$

Now by Eq. (22) and Eq. (23), we have

$$
\left\|U_{T, 1}^{N}\right\| \leq\left\|I_{T, N} \frac{d}{d t} Y-\frac{d}{d t} Y\right\|_{T}+\left\|\frac{d}{d t}\left(I_{T, N} Y-Y\right)\right\|_{T} \leq c T N^{-r} R_{T, 1}^{r}(Y)
$$

Here $2 c_{1}=c$, which completes the proof.

Now, to analyze the numerical error of functions $f$ and $g$, we consider the following, for a positive number $\beta<1 / 4$.

Theorem 3.1 ([27]) If conditions Eq. (2)-Eq. (5) hold, $R_{T, 1}^{r}(\Phi)$ is a finite function for integers $1 \leq r \leq N$, and

$$
T \sqrt{2+N^{-1}}\left(1+\sqrt{2+N^{-1}}\right)\left(K_{1}+K_{2}(1-\lambda)\right) \leq \beta<\frac{1}{4},
$$


where $0 \leq \lambda<1$, then

$$
\begin{aligned}
& \left\|Y-y^{N}\right\|_{T} \leq c_{\beta} T^{2} N^{-r} R_{T, 1}^{r}(Y), \\
& \left|Y(T)-y^{N}(T)\right| \leq c_{\beta} T^{\frac{3}{2}} N^{-r} R_{T, 1}^{r}(Y),
\end{aligned}
$$

and, in particular,

$$
\max _{t \in[0, T]}\left|Y(t)-y^{N}(t)\right| \leq c_{\beta} T N^{1-r} R_{T, 1}^{r}(Y)
$$

where the constant $c_{\beta}>0$, depending on $\beta$.

Proof Since $U_{T, 2}^{N}(0)=0$, we have from Eq. (15)

$$
\begin{aligned}
\left\|U_{T, 2}^{N}\right\|_{T, N}= & \left\|f\left(y^{N}, v_{1}^{N}, \cdot\right)-f\left(I_{T, N} Y, I_{T, N} V_{1}, \cdot\right)\right\|_{T, N} \\
& \vee\left\|g\left(y^{N}, v_{2}^{N}, \cdot\right)-g\left(I_{T, N} Y, I_{T, N} V_{2}, \cdot\right)\right\|_{T, N}, \quad t \in \Lambda_{N},
\end{aligned}
$$

where $v_{1}^{N}(t)=\int_{0}^{t_{i}} G(t, s) y^{N}(s) d s, v_{2}^{N}(t)=\int_{0}^{t_{i}} H(t, s) y^{N}(s) d s \in P_{N}(0, T)$. For simplicity, we denote $K_{N}=\sqrt{2+N^{-1}}$, then, by using Eq. (2) and Eq. (3), we see that Eq. (29) takes the form

$$
\begin{aligned}
\left\|U_{T, 2}^{N}\right\|_{T, N} \leq & \left\|f\left(y^{N}, v_{1}^{N}, \cdot\right)-f\left(I_{T, N} Y, v_{1}^{N}, \cdot\right)\right\|_{T, N} \\
& +\left\|f\left(I_{T, N} Y, v_{1}^{N}, \cdot\right)-f\left(I_{T, N} Y, I_{T, N} V_{1}, \cdot\right)\right\|_{T, N} \\
& \vee\left\|g\left(y^{N}, v_{1}^{N}, \cdot\right)-g\left(I_{T, N} Y, v_{1}^{N}, \cdot\right)\right\|_{T, N} \\
& +\left\|g\left(I_{T, N} Y, v_{1}^{N}, \cdot\right)-g\left(I_{T, N} Y, I_{T, N} V_{1}, \cdot\right)\right\|_{T, N} \\
\leq & K_{1}\left\|E^{N}\right\|_{T, N}+K_{2}\left\|v^{N}-I_{T, N} V\right\|_{T, N} \\
\leq & K_{N}\left(K_{1}\left\|E^{N}\right\|_{T}+K_{2}\left\|v^{N}-I_{T, N} V\right\|_{T}\right) .
\end{aligned}
$$

Furthermore, using Eq. (21) we obtain

$$
\begin{aligned}
\left\|v^{N}-I_{T, N} V\right\|_{T} & \leq\left\|v^{N}-V\right\|_{T}+\left\|V-I_{T, N} V\right\|_{T} \\
& \leq(1-\lambda)^{-\frac{1}{2}}\left\|Y-y^{N}\right\|_{T}+c T^{2} N^{-r-1} R_{T, 1}^{r}(Y) .
\end{aligned}
$$

Now by Eq. (25), Eq. (30), and Eq. (31), we get

$$
\left\|U_{T, 2}^{N}\right\|_{T, N} \leq K_{N}\left(K_{1}\left\|E^{N}\right\|_{T}+K_{2}(1-\lambda)^{-\frac{1}{2}}\left\|Y-y^{N}\right\|_{T}\right)+c K_{N} K_{2} T^{2} N^{-r-1} R_{T, 1}^{r}(Y) .
$$

Now for any $t \in[0, T]$ (cf. [28])

$$
\left(E^{N}(t)\right)^{2} \leq\left(T^{\frac{1}{2}}\left(E^{N}(T)\right)^{2}+2\left\|E^{N}\right\|_{T}\left\|t^{\frac{1}{2}} \frac{d}{d t} E^{N}\right\|_{T}\right) t^{-\frac{1}{2}} .
$$

Integrating Eq. (33) with respect to $t$,

$$
\left\|E^{N}\right\|_{T}^{2} \leq 2 T\left(E^{N}(T)\right)^{2}+4 T^{\frac{1}{2}}\left\|E^{N}\right\|_{T}\left\|t^{\frac{1}{2}} \frac{d}{d t} E^{N}\right\|_{T} .
$$


Now since $\frac{d}{d t} E^{N}(t) \in P_{N-1}(0, T)$ and $\left|U_{T, 1}^{N}(0)+U_{T, 2}^{N}(0)\right|<\infty$, we have

$$
\begin{aligned}
\left\|t^{\frac{1}{2}} \frac{d}{d t} E^{N}\right\|_{T} & =\left\|t^{\frac{1}{2}} \frac{d}{d t} E^{N}\right\|_{T, N}=\left\|t^{\frac{1}{2}}\left(U_{T, 1}^{N}+U_{T, 2}^{N}\right)\right\|_{T, N} \\
& \leq T^{\frac{1}{2}}\left\|U_{T, 1}^{N}\right\|_{T, N}+T^{\frac{1}{2}}\left\|U_{T, 2}^{N}\right\|_{T, N^{*}}
\end{aligned}
$$

We use Eq. (34) and Eq. (35) to derive that

$$
\left\|E^{N}\right\|_{T}^{2} \leq 2 T\left(E^{N}(T)\right)^{2}+4 T\left\|E^{N}\right\|_{T}\left(\left\|W_{T, 1}^{N}\right\|_{T, N}+\left\|W_{T, 2}^{N}\right\|_{T, N}\right)
$$

which implies

$$
\left(E^{N}(T)\right)^{2} \geq \frac{1}{2 T}\left\|E^{N}\right\|_{T}^{2}-2\left\|E^{N}\right\|_{T}\left(\left\|U_{T, 1}^{N}\right\|_{T, N}+\left\|U_{T, 2}^{N}\right\|_{T, N}\right) .
$$

Combining Eq. (18) and Eq. (37) gives

$$
\begin{aligned}
& \frac{1}{2 T}\left\|E^{N}\right\|_{T}^{2}-2\left\|E^{N}\right\|_{T}\left(\left\|U_{T, 1}^{N}\right\|_{T, N}+\left\|U_{T, 2}^{N}\right\|_{T, N}\right) \\
& \quad \leq 2\left\|E^{N}\right\|_{T, N}\left\|U_{T, 1}^{N}\right\|_{T, N}+2\left\|E^{N}\right\|_{T, N}\left\|U_{T, 2}^{N}\right\|_{T, N},
\end{aligned}
$$

which together with Eq. (21), Eq. (25) and Eq. (32) yields

$$
\begin{aligned}
\left\|E^{N}\right\|_{T} & \leq 4 T\left(1+K_{N}\right)\left(\left\|U_{T, 1}^{N}\right\|_{T, N}+\left\|U_{T, 2}^{N}\right\|_{T, N}\right) \\
& \leq 4 T K_{N}\left(1+K_{N}\right)\left(K_{1}\left\|E^{N}\right\|_{T}+K_{2}(1-\lambda)^{-\frac{1}{2}}\left\|Y-y^{N}\right\|_{T}\right)+c T^{2} N^{-r} R_{T, 1}^{r}(Y) .
\end{aligned}
$$

Here

$$
\left\|Y-y^{N}\right\|_{T} \leq\left\|E^{N}\right\|_{T}+\left\|Y-I_{T, N} Y\right\|_{T} \leq+\left\|E^{N}\right\|_{T}+c T^{2} N^{-r-1} R_{T, 1}^{r}(Y) .
$$

Putting Eq. (40) into Eq. (39), we have

$$
\begin{aligned}
& \left(1-4 T K_{N}\left(1+K_{N}\right)\left(K_{1}+K_{2}(1-\lambda)^{-\frac{1}{2}}\right)\right)\left\|E^{N}\right\|_{T} \\
& \quad \leq c K_{2} K_{N}\left(1+K_{N}\right)(1-\lambda)^{-\frac{1}{2}} T^{3} N^{-r-1} R_{T, 1}^{r}(Y)+c T^{2} N^{-r} R_{T, 1}^{r}(Y) \\
& \quad \leq c T^{2} N^{-r} R_{T, 1}^{r}(Y),
\end{aligned}
$$

combining Eq. (41) with Eq. (25), we have

$$
\left\|E^{N}\right\|_{T} \leq c_{\beta} T^{2} N^{-r} R_{T, 1}^{r}(Y)
$$

Equation (42) with Eq. (40) gives

$$
\left\|Y-y^{N}\right\|_{T} \leq c_{\beta} T^{2} N^{-r} R_{T, 1}^{r}(Y)
$$

which leads to Eq. (26). 
Next by using Eq. (32), Eq. (42), and Eq. (43), we have

$$
\left\|U_{T, 2}^{N}\right\|_{T, N} \leq c_{\beta} T^{2} N^{-r} R_{T, 1}^{r}(Y)
$$

Furthermore, using Eq. (18), Eq. (21), Eq. (42), and Eq. (44), we deduce that

$$
\begin{aligned}
\left|Y(T)-y^{N}(T)\right|^{2} & =\left(E^{N}(T)\right)^{2} \leq 2 K_{N}\left\|E^{N}\right\|_{T}\left(\left\|U_{T, 1}^{N}\right\|_{T, N}+\left\|U_{T, 2}^{N}\right\|_{T, N}\right) \\
& \leq c_{\beta} T^{3} N^{-2 r}\left(R_{T, 1}^{r}(Y)\right)^{2} .
\end{aligned}
$$

This proves Eq. (27).

Again using Eq. (23), and Eq. (42), we deduce that

$$
\begin{aligned}
\left\|\frac{d}{d t}\left(Y-y^{N}\right)\right\|_{T} & \leq\left\|\frac{d}{d t} E^{N}\right\|_{T}+\left\|\frac{d}{d t}\left(Y-I_{T, N} Y\right)\right\|_{T} \\
& \leq c T^{-1} N^{2}\left\|E^{N}\right\|_{T}+\left\|\frac{d}{d t}\left(Y-I_{T, N} Y\right)\right\|_{T} \\
& \leq c_{\beta} T N^{2-r} R_{T, 1}^{r}(Y)+c T N^{-r} R_{T, 1}^{r}(Y) \\
& \leq c_{\beta} T N^{2-r} R_{T, 1}^{r}(Y) .
\end{aligned}
$$

This together with Eq. (43) implies that

$$
\left\|Y-y^{N}\right\|_{H^{1}(0, T)} \leq c_{\beta} T N^{2-r} R_{T, 1}^{r}(Y)
$$

For this purpose, according to the Sobolev inequality [26],

$$
\|u\|_{L^{\infty}(0, T)} \leq \sqrt{2+T^{-1}}\|u\|_{L^{2}(0, T)}^{\frac{1}{2}}\|u\|_{H^{1}(0, T)}^{\frac{1}{2}} .
$$

Hence by Eq. (26) and Eq. (46), we obtain

$$
\begin{aligned}
\max _{t \in[0, T]}\left|Y(t)-y^{N}(t)\right| & \leq \sqrt{2+T^{-1}}\left\|Y-y^{N}\right\|_{L^{2}(0, T)}^{\frac{1}{2}}\left\|Y-y^{N}\right\|_{H^{1}(0, T)}^{\frac{1}{2}} \\
& \leq c_{\beta} T N^{1-r} R_{T, 1}^{r}(Y)
\end{aligned}
$$

which is the proof of Eq. (28).

\section{Numerical results}

In this section, we perform a number of numerical experiments to check the efficiency of the proposed scheme. In our simulations we use Legendre-Gauss-Lobatto points with the following norms:

$$
\begin{aligned}
L^{\infty} & =\max \left|\left(\phi^{N}(t)-\phi(t)\right)\right| ; \\
L^{1} & =\frac{1}{2}\left|\left(\phi^{N}(t)-\phi(t)\right)\right|^{\prime} w ; \\
L^{2} & =\sqrt{\frac{1}{2}\left|\left(\phi^{N}(t)-\phi(t)\right)\right|^{2}} w ;
\end{aligned}
$$


Table 1 The point-wise error using Eq. (13) of Eq. (51)

\begin{tabular}{rlll}
\hline$N$ & $L^{\infty}$-norm & $L^{1}$-norm & $L^{2}$-norm \\
\hline 2 & $3.450 \mathrm{e}-02$ & $1.754 \mathrm{e}-02$ & $2.175 \mathrm{e}-02$ \\
8 & $1.224 \mathrm{e}-02$ & $7.038 \mathrm{e}-03$ & $8.380 \mathrm{e}-03$ \\
14 & $6.028 \mathrm{e}-03$ & $3.271 \mathrm{e}-03$ & $3.877 \mathrm{e}-03$ \\
20 & $3.441 \mathrm{e}-03$ & $1.583 \mathrm{e}-03$ & $1.993 \mathrm{e}-03$ \\
\hline
\end{tabular}

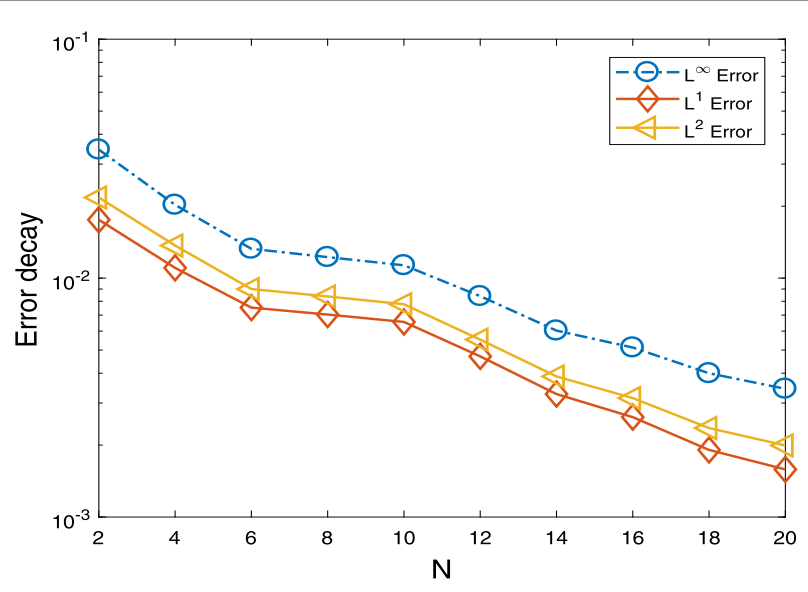

Figure 1 Numerical errors of Eq. (51) at $T=1$

where $y^{N}(t)$ is the global numerical solution. We apply the present method for numerical solutions and compare with exact solutions of sample problems. For computations, we use the final time $T=1$ and $N=20$ and the Legendre-Gauss quadrature with weights:

$$
\omega_{m}=\left(\frac{2}{\left(1-x_{m}^{2}\right)\left[L_{N+1}^{\prime}\left(x_{m}\right)\right]^{2}}\right), \quad 0<m<N .
$$

Example 4.1 ([29]) Consider the following SVIDE:

$$
y(t)=y_{0}+\int_{0}^{t} s^{2} y(s) d s+\int_{0}^{t} s y(s) d W(s), \quad \forall t, s \in[0, T]
$$

the initial condition is $y(0)=1$, actual solution of Eq. (51) is

$$
y(t)=\exp \left(\frac{t^{3}}{6}+\int_{0}^{t} s d W(s)\right)
$$

where $y(t)$ is an unknown stochastic process, and $W(t)$ is a Brownian motion process. The errors with different norms between numerical solution by the spectral method and the exact solution are shown in Table 1 and Fig. 1, which shows the efficiency of our proposed scheme. The comparison of exact and numerical solutions is shown in Fig. 2, which confirms their good agreement.

Example 4.2 ([29]) Consider the SVIDE,

$$
y(t)=y_{0}+\int_{0}^{t} \cos (s) y(s) d s+\int_{0}^{t} \sin (s) y(s) d W(s), \quad t, s \in[0, T],
$$




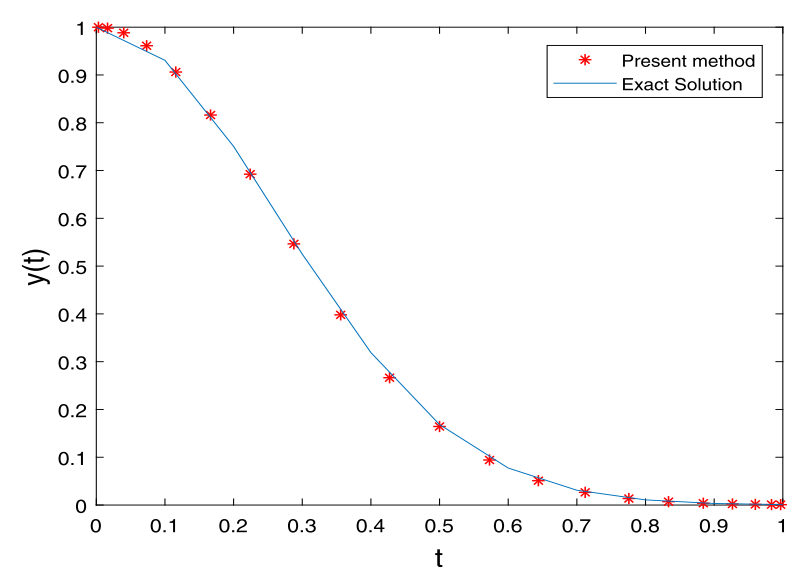

Figure 2 Comparison of exact and approximate solution of Example 4.1

Table 2 The point-wise error using Eq. (13) of Eq. (52)

\begin{tabular}{rlll}
\hline$N$ & $L^{\infty}$-norm & $L^{1}$-norm & $L^{2}$-norm \\
\hline 2 & $4.678 \mathrm{e}-03$ & $4.678 \mathrm{e}-03$ & $2.763 \mathrm{e}-03$ \\
8 & $1.658 \mathrm{e}-03$ & $9.406 \mathrm{e}-04$ & $1.141 \mathrm{e}-03$ \\
14 & $8.092 \mathrm{e}-04$ & $4.341 \mathrm{e}-04$ & $5.153 \mathrm{e}-04$ \\
20 & $4.328 \mathrm{e}-04$ & $2.033 \mathrm{e}-04$ & $2.525 \mathrm{e}-04$ \\
\hline
\end{tabular}

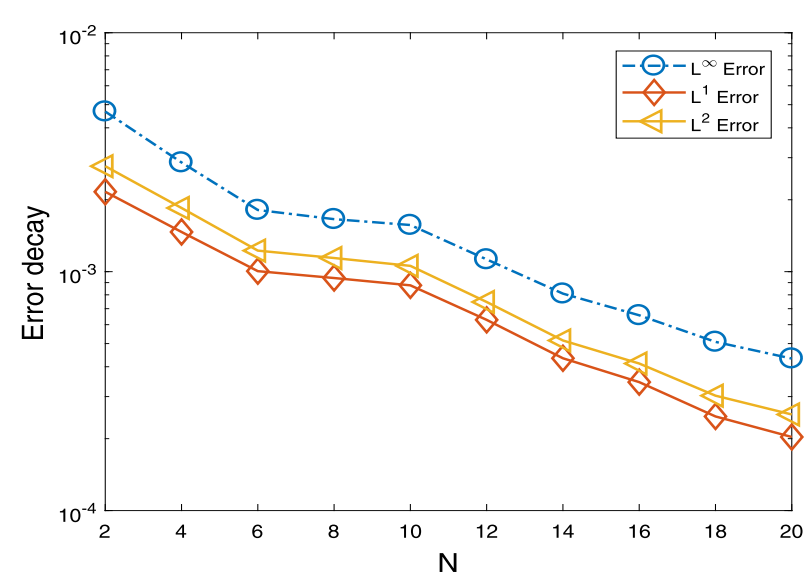

Figure 3 Numerical errors of Eq. (52) at $T=1$

where $y(t)$ is an unknown stochastic process, and $W(t)$ is a Brownian motion process. The initial condition is $y(0)=\frac{1}{12}$, and the exact solution of Eq. (52) is given by

$$
y(t)=\frac{1}{12} \exp \left(\frac{-t}{4}+\sin (t)+\frac{\sin (2 t)}{8}+\int_{0}^{t} \sin (s) d W(s)\right) .
$$

The different norms are estimated for different $N$, between the exact and numerical solutions as shown in Table 2 and Fig. 3. The comparison of exact and numerical approximations is shown in Fig. 4. 


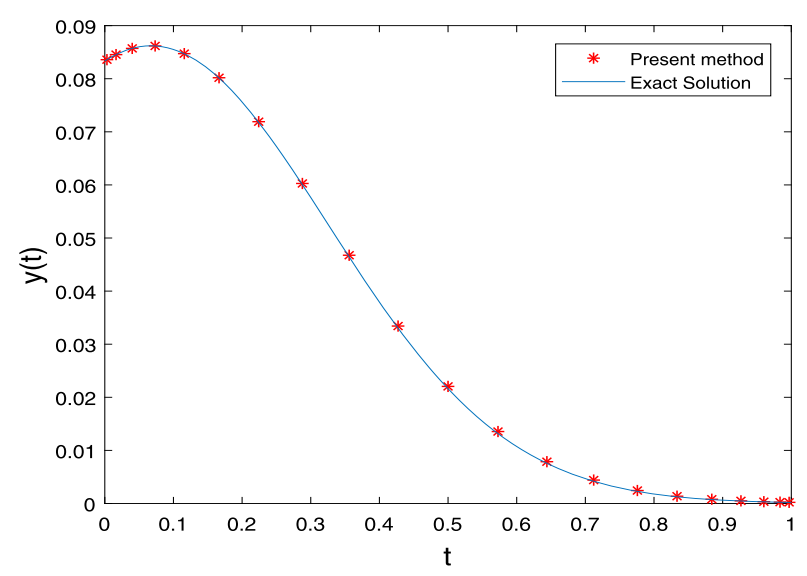

Figure 4 Comparison of exact and approximate solution of Example 4.2

Table 3 The point-wise error using Eq. (13) of Eq. (53)

\begin{tabular}{rlll}
\hline$N$ & $L^{\infty}$-norm & $L^{1}$-norm & $L^{2}$-norm \\
\hline 2 & $2.952 \mathrm{e}-03$ & $1.606 \mathrm{e}-03$ & $1.841 \mathrm{e}-03$ \\
8 & $3.034 \mathrm{e}-05$ & $1.212 \mathrm{e}-05$ & $1.555 \mathrm{e}-05$ \\
14 & $9.747 \mathrm{e}-06$ & $2.620 \mathrm{e}-06$ & $4.258 \mathrm{e}-06$ \\
20 & $3.685 \mathrm{e}-06$ & $7.545 \mathrm{e}-07$ & $1.410 \mathrm{e}-06$ \\
\hline
\end{tabular}

Example 4.3 ([30]) Consider the linear SVIDE,

$$
y(t)=y_{0}+\int_{0}^{t} u(s) y(s) d s+\sum_{i=1}^{m} \int_{0}^{t} \beta_{i}(s) y(s) d W_{i}(s), \quad t, s \in[0, T],
$$

where $y(t)$ is an unknown stochastic process defined on a complete probability space, and $W(t)=\left(W_{1}(t), W_{2}(t), \ldots, W_{m}(t)\right)$ is an $m$-dimension Brownian motion process, subject to initial condition $y(0)=\frac{1}{12}$. The exact solution of Eq. (53) is

$$
y(t)=\frac{1}{12} \exp \left(\int_{0}^{t}\left(u(s)-\frac{1}{2} \sum_{i=1}^{m} \beta_{i}^{2}(s)\right) d s+\sum_{i=1}^{m} \int_{0}^{t} \beta_{i}(s) d W_{i}(s)\right) .
$$

For the numerical simulation we choose $m=3, u(s)=s^{2}, \beta_{1}(s)=\sin (s), \beta_{2}(s)=\cos (s)$ and $\beta_{3}(s)=s$. The errors in different norms between the exact solutions and numerical solutions are shown in Table 3 and Fig. 5, which confirms the efficiency of the present method. The comparison of exact and numerical solutions of Eq. (53) is shown in Fig. 6.

\section{Conclusion}

In this article, a Legendre-spectral method with Legendre-Gauss-Lobotto points as collocation is presented for stochastic integro-differential equations. A comprehensive error analysis of the scheme is provided and a number of numerical experiments were performed to validate the theoretical results. Both our theoretical and numerical results show that our numerical method has a spectral rate of convergence which is a maximum that any numerical method can achieve. In the future this scheme can be generalized to nonlinear SVIDEs, and a system of both linear and non-linear SVIDEs. 


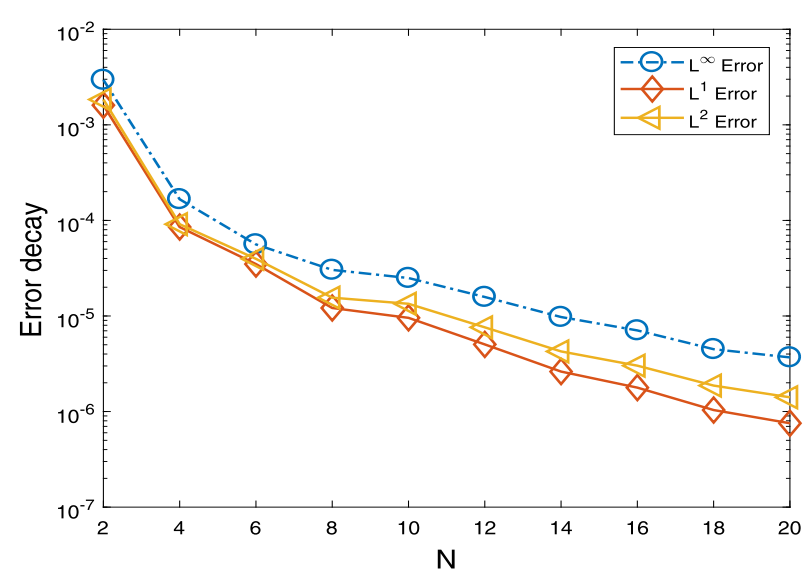

Figure 5 Numerical errors of Eq. (53) at $T=1$

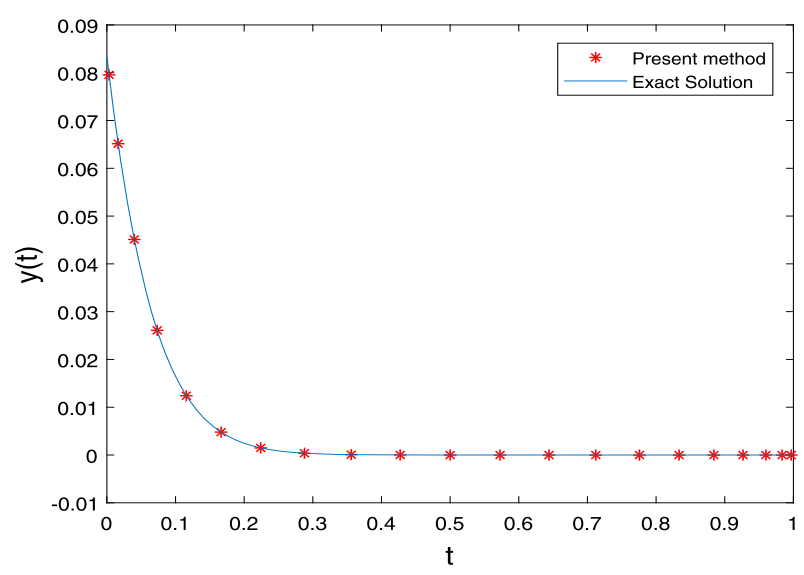

Figure 6 Comparison of exact and approximate solution of Example 4.3

Acknowledgements

Not applicable.

\section{Funding}

No funding available.

\section{Availability of data and materials}

Not applicable.

Ethics approval and consent to participate

Not applicable.

\section{Competing interests}

The authors declare that they have no competing interests.

\section{Consent for publication}

Not applicable.

Authors' contributions

All authors equally contributed in the preparation of this manuscript. All authors read and approved the final manuscript.

\section{Author details}

'Department of Mathematics, COMSATS University, Islamabad, Pakistan. ${ }^{2}$ Department of Physics, COMSATS University, Islamabad, Pakistan. ${ }^{3}$ Department of Mathematics and Statistics, College of Science, King Faisal University, Al-Ahsa, Kingdom of Saudi Arabia. 


\section{Publisher's Note}

Springer Nature remains neutral with regard to jurisdictional claims in published maps and institutional affiliations.

\section{Received: 30 November 2018 Accepted: 12 April 2019 Published online: 27 April 2019}

\section{References}

1. Levin, J.J., Nohel, J.A.: On a system of integro-differential equations occurring in reactor dynamics. J. Math. Mech. 9, 347-368 (1960)

2. Miller, R.K.: On a system of integro-differential equations occurring in reactor dynamics. SIAM J. Appl. Math. 14, 446-452 (1966)

3. Cioica, P.A., Dahlke, S.: Spatial Besov regularity for semi linear stochastic partial differential equations on bounded Lipschitz domains. Int. J. Comput. Math. 89(18), 2443-2459 (2012)

4. Khodabin, M., Maleknejad, K., Rostami, M., Nouri, M.: Interpolation solution in generalized stochastic exponential population growth model. Appl. Math. Model. 36, 1023-1033 (2012)

5. Oguztöreli, M.N.: Time-Lag Control Systems. Academic Press, New York (1966)

6. Maleknejad, K., Khodabin, M., Rostami, M.: Numerical solutions of stochastic Volterra integral equations by a stochastic operational matrix based on Bloch pulse functions. Math. Comput. Model. 55, 791-800 (2012)

7. Khodabin, M., Maleknejad, K., Rostami, M., Nouri, M.: Numerical approach for solving stochastic Volterra-Fredholm integral equations by stochastic operational matrix. Comput. Math. Appl. 64, 1903-1913 (2012)

8. Khodabin, M., Maleknejad, K., Rostami, M., Nouri, M.: Numerical solution of stochastic differential equations by second order Runge-Kutta methods. Appl. Math. Model. 53, 1910-1920 (2011)

9. Berger, M., Mizel, V.: Volterra equations with Itô integrals, I. J. Integral Equ. 2, 187-245 (1980)

10. Ali, I., Brunner, H., Tang, T.: A spectral method for pantograph-type delay differential equations and its convergence analysis. J. Comput. Math. 27, 254-265 (2009)

11. Ali, I., Brunner, H., Tang, T.: Spectral methods for pantograph-type differential and integral equations with multiple delays. Front. Math. China 4, 49-61 (2009)

12. Khan, S.U., Ali, I.: Application of Legendre spectral-collocation method to delay differential and stochastic delay differential equation. AlP Adv. 8(3), 035301 (2018)

13. Cardone, A., D'Ambrosio, R., Paternoster, B.: A spectral method for stochastic fractional differential equations. Appl. Numer. Math. 139, 115-119 (2019)

14. Cardone, A., Conte, D., D'Ambrosio, R., Paternoster, B.: Stability issues for selected stochastic evolutionary problems: a review. Axioms 7(4), 91 (2018)

15. Conte, D., D'Ambrosio, R., Paternoster, B.: On the stability of theta-methods for stochastic Volterra integral equations. Discrete Contin. Dyn. Syst., Ser. B (2018). https://doi.org/10.3934/dcdsb.2018087

16. Burrage, K., Cardone, A., D'Ambrosio, R., Paternoster, B.: Numerical solution of time fractional diffusion systems. Appl. Numer. Math. 116, 82-94 (2017)

17. Oksendal, B.: Stochastic Differential Equations, an Introduction with Applications, 5th edn. Springer, New York (1998)

18. Hu, P., Huang, C.: The stochastic $\theta$-method for nonlinear stochastic Volterra integro-differential equations. Abstr. Appl. Anal. 2014, Article ID 583930 (2014)

19. Buckwar, E., Winkler, R.: Multistep method for SDEs and their application to problem with small noise. SIAM J. Numer. Anal. 44(2), 779-803 (2006)

20. Tian, T.H., Burrage, K.: Implicit Taylor method for stiff stochastic differential equations. Appl. Numer. Math. 38(1-2), 167-185 (2001)

21. Golec, J., Sathannathan, S.: Strong approximation of stochastic integro differential equations. Dyn. Contin. Discrete Impuls. Syst., Ser. B, Appl. Algorithms 8(1), 139-151 (2001)

22. Shaikhet, L.E., Roberts, J.A.: Reliability of difference analogues to preserve the stability properties stochastic Volterra integro-differential equations. Adv. Differ. Equ. 2006, Article ID 73897 (2006)

23. Ali, l.: Convergence analysis of spectral methods for integro-differential equations with vanishing proportional delays. J. Comput. Math. 29(1), 50-61 (2011)

24. Canuto, C., Hussaini, M., Quarteroni, A., Zang, T.A.: Spectral Methods: Fundamental in Single Domains. Springer, Berlin (2006)

25. Shen, J., Tang, T.: Spectral and High-Order Method with Applications. Science Press, Beijing (2006)

26. Li-jun, Y., Zi-qiang, L., Zhong-qing, W.: Legendre-Gauss-Lobatto spectral collocation method for nonlinear delay differential equations. Math. Methods Appl. Sci. 36(18), 2476-2491 (2013)

27. Yi, L., Wang, Z.: A Legendre-Gauss-Radau spectral collocation method for first order nonlinear delay differential equations. Calcolo (2015). https://doi.org/10.1007/s10092-015-0169-5

28. Guo, B.Y., Wang, Z.Q.: A spectral collocation method for solving initial value problems of first order ordinary differential equations. Discrete Contin. Dyn. Syst., Ser. B 14, 1029-1054 (2010)

29. Mohammadi, F.: Numerical solution of stochastic Itô-Volterra integral equations using Haar wavelets. Numer. Math., Theory Methods Appl. 9(3), 416-431 (2016)

30. Maleknejad, K., Khodabin, M., Rostami, M.: A numerical method for solving m-dimensional stochastic Itô-Volterra integral equations by stochastic operational matrix. Comput. Math. Appl. 63, 133-143 (2012) 